\title{
$\begin{array}{lllllllll}\mathrm{I} & \mathrm{N} & \mathrm{S} & \mathrm{T} & \mathrm{I} & \mathrm{T} & \mathrm{U} & \mathrm{T} & \mathrm{E}\end{array}$
}

\section{Out-of-Home Care by State and Place: Higher Placement Rates for Children in Some Remote Rural Places}

\author{
MARYBETH J. MATTINGLY, MELISSA WELLS, AND MICHAEL DINEEN
}

$\mathrm{T}$ The lives of children placed in out-of-home care as a result of abuse or neglect are severely disrupted. Children are generally removed from their homes only as a last resort when their or others' safety and well-being are at risk. These placements aim to alleviate distress and improve child wellbeing. However, it is often difficult for policy makers to fully understand variations in placement rates by location within their states. In this fact sheet, we show breakdowns by metropolitan status for the nation, regions, and states in hopes that the information will be useful as changes to and funding for the child welfare system are considered.

Counties are classified according to the U.S. Department of Agriculture's rural-urban continuum codes. This approach contrasts placement rates in metropolitan areas, termed "metropolitan/urban," with nonmetro counties adjacent to metropolitan areas, termed "adjacent nonmetro," and those not adjacent to metropolitan areas, termed "remote rural."

Table 1 shows rates (per 1,000) of children and youth in outof-home care at any time in 2007 by place, state, and region. Nationally and within each region, remote rural areas have higher rates of out-of-home placement. Additionally, nearly half of the states have the highest placement rates in remote rural areas.

There are several potential reasons for observed differences. Larger populations of African American or Latino children who tend to have higher placement rates may increase the overall risk of placement in the area, particularly in urban areas. ${ }^{2}$ Higher poverty rates in some states or regions may increase the need for child welfare resources, including out-of-home placement. A scarcity of supportive services in rural areas or gaps in mental health services to address issues contributing to out-of-home placements may lead to higher placement rates in resource-poor areas. ${ }^{3}$ Finally, differences in child welfare agencies' policies and procedures might result in differing placement rates across states and types of communities.

\section{Data}

We used Adoption and Foster Care Reporting System (AFCARS) data. ${ }^{4}$ AFCARS data are collected at regular intervals in every state and reflect all placements, providing "24-hour substitute care for children outside their own homes."5 State-level differences may contribute to variations in types of placements included in AFCARS.

\section{About the Authors}

Marybeth J. Mattingly is director of research on vulnerable families at the Carsey Institute (beth.mattingly@unh.edu).

Melissa Wells is an assistant professor of social work and a faculty fellow at the Carsey Institute (melissa.wells@unh.edu).

Michael Dineen is a data analyst at the National Data Archive on Child Abuse and Neglect at Cornell University (med39@cornell.edu).

\section{ACKNOWLEDGMENTS}

The authors thank Michelle Stransky and Kai Ji for their assistance in preparing this brief. They also thank Jean Dawson, Lisa Jones, and Christian Connell for thoughtful comments and suggestions.

\section{ENDNOTES}

1. See http://www.ers.usda.gov/briefing/Rurality/ RuralUrbCon/.

2. Fred Wulczyn and Kristin Brunner Hislop, "Topic \#1: Foster Care Dynamics in Urban and Non-Urban Counties" (Chicago: Chapin Hall Center for Children, 2003).

3. Kathleen Belanger and Warren Stone, "The Social Service Divide: Service Availability in Rural Versus Urban Counties and Impact on Child Welfare Outcomes," Child Welfare 87 (2008): 101-124; Richard P. Barth, Judy Wildfire, and Rebecca L. Green, "Placement into Foster Care and the Interplay of Urbanicity, Child Behavior Problems, and Poverty," American Journal of Orthopsychiatry 76 (2006): 358-366.

4. The data used in this publication were made available by the National Data Archive on Child Abuse and Neglect, Cornell University in Ithaca, New York, and have been used with permission. Data from the AFCARS were originally collected by the Children's Bureau.

5. Child Welfare Information Gateway, "Foster Care Statistics: Numbers and Trends."

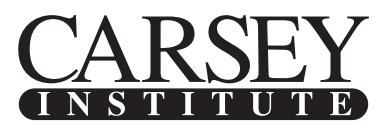

BUILDING KNOWLEDGE FOR Families ANd Communities IN THE 21ST CENTURY
Huddleston Hall, 73 Main Street, Durham, NH 03824

(603) 862-2821 • www.carseyinstitute.unh.edu

This work is supported by the Children's Bureau, Administration on Children, Youth and Families, Administration for Children and Families, U.S. Department of Health and Human Services. 
TABle 1. OUT-OF-HOME PLACEMENT DURING 2007 BY STATE AND PLACE

\begin{tabular}{|c|c|c|c|c|c|c|c|c|}
\hline & \multicolumn{2}{|c|}{ METROPOLITAN } & \multicolumn{2}{|c|}{ Nonmetro AdJACENT } & \multicolumn{2}{|c|}{ REMOTE RURAL } & \multicolumn{2}{|c|}{ Total } \\
\hline & $\begin{array}{c}\text { Rate } \\
(\text { per } 1,000)\end{array}$ & $\begin{array}{c}\text { Population } \\
\text { Served }\end{array}$ & $\begin{array}{c}\text { Rate } \\
(\text { per } 1,000)\end{array}$ & $\begin{array}{c}\text { Population } \\
\text { Served }\end{array}$ & $\begin{array}{c}\text { Rate } \\
\text { (per } 1,000)\end{array}$ & $\begin{array}{c}\text { Population } \\
\text { Served }\end{array}$ & $\begin{array}{c}\text { Rate } \\
\text { (per } 1,000)\end{array}$ & $\begin{array}{c}\text { Population } \\
\text { Served }\end{array}$ \\
\hline United States & 10.3 & 641,359 & 10.8 & 82,766 & 13.7 & 53,273 & 10.5 & 777,398 \\
\hline Northeast & 10.4 & 118,153 & 10.0 & 9,151 & 11.9 & 2,461 & 10.4 & 129,765 \\
\hline Midwest & 10.8 & 136,799 & 10.3 & 21,812 & 14.8 & 21,150 & 11.1 & 179,761 \\
\hline South & 8.9 & 196,612 & 10.7 & 39,917 & 12.2 & 18,115 & 9.3 & 254,644 \\
\hline West & 11.7 & 189,795 & 13.1 & 11,886 & 14.8 & 11,547 & 12.0 & 213,228 \\
\hline Alabama & 10.2 & 8,192 & 9.1 & 2,515 & 5.1 & 197 & 9.7 & 10,904 \\
\hline Alaska & 14.3 & 1,750 & 7.6 & 30 & 21.0 & 1,167 & 16.2 & 2,947 \\
\hline Arizona & 10.3 & 15,514 & 8.6 & 1,439 & NA & - & 10.1 & 16,953 \\
\hline Arkansas & 9.9 & 4,262 & 11.4 & 1,544 & 12.1 & 1,598 & 10.6 & 7,404 \\
\hline California & 12.3 & 113,321 & 18.7 & 2,231 & 18.8 & 966 & 12.4 & 116,518 \\
\hline Colorado & 11.9 & 12,355 & 18.7 & 932 & 11.0 & 1,075 & 12.1 & 14,362 \\
\hline Connecticut & 10.8 & 8,121 & 12.1 & 813 & NA & - & 10.9 & 8,934 \\
\hline Delaware & 10.6 & 1,748 & 8.7 & 347 & $\mathrm{NA}$ & - & 10.2 & 2,095 \\
\hline District of Columbia & 26.0 & 2,943 & $\mathrm{NA}$ & - & $\mathrm{NA}$ & - & 26.0 & 2,943 \\
\hline Florida & 11.4 & 43,288 & 17.2 & 4,041 & $\mathrm{NA}$ & - & 11.8 & 47,329 \\
\hline Georgia & 7.9 & 16,377 & 12.0 & 4,285 & 13.2 & 1,180 & 8.7 & 21,842 \\
\hline Hawaii & 12.5 & 2,511 & NA & - & 14.1 & 1,214 & 13.0 & 3,725 \\
\hline Idaho & 8.4 & 2,277 & 6.6 & 513 & 9.8 & 564 & 8.2 & 3,354 \\
\hline Illinois & 7.4 & 20,802 & 5.6 & 1,211 & 12.1 & 1,772 & 7.5 & 23,785 \\
\hline Indiana & 12.1 & 15,002 & 11.0 & 3,164 & 12.0 & 633 & 11.9 & 18,799 \\
\hline Iowa & 19.9 & 8,137 & 14.3 & 2,306 & 22.6 & 3,176 & 19.1 & 13,619 \\
\hline Kansas & 13.6 & 6,196 & 19.6 & 1,429 & 15.3 & 2,589 & 14.6 & 10,214 \\
\hline Kentucky & 12.1 & 7,169 & 11.6 & 2,067 & 16.0 & 3,732 & 12.9 & 12,968 \\
\hline Louisiana & 7.6 & 6,124 & 8.7 & 2,332 & 7.3 & 185 & 7.8 & 8,641 \\
\hline Maine & 10.7 & 1,792 & 7.4 & 615 & 16.9 & 492 & 10.4 & 2,899 \\
\hline Maryland & 9.6 & 12,316 & 6.3 & 426 & NA & - & 9.4 & 12,742 \\
\hline Massachusetts & 11.7 & 16,699 & NA & - & NA & - & 11.6 & 16,699 \\
\hline Michigan & 12.3 & 24,923 & 10.5 & 2,126 & 11.3 & 2,325 & 12.0 & 29,374 \\
\hline Minnesota & 10.4 & 9,784 & 13.8 & 2,287 & 14.3 & 2,165 & 11.3 & 14,236 \\
\hline Mississippi & 7.2 & 2,470 & 6.3 & 932 & 7.2 & 1,969 & 7.0 & 5,371 \\
\hline Missouri & 9.7 & 10,270 & 12.5 & 2,212 & 13.5 & 2,532 & 10.5 & 15,014 \\
\hline Montana & 13.6 & 1,064 & 14.7 & 440 & 13.0 & 1,456 & 13.5 & 2,960 \\
\hline Nebraska & 21.7 & 5,806 & 29.1 & 872 & 19.7 & 2,901 & 21.5 & 9,579 \\
\hline Nevada & 13.7 & 8,164 & 8.3 & 331 & 9.2 & 211 & 13.2 & 8,706 \\
\hline New Hampshire & 4.9 & 959 & 6.0 & 422 & 9.2 & 294 & 5.6 & 1,675 \\
\hline New Jersey & 7.8 & 16,075 & NA & - & NA & - & 7.8 & 16,075 \\
\hline New Mexico & 7.4 & 2,429 & 10.0 & 817 & 14.6 & 1,330 & 9.1 & 4,576 \\
\hline New York & 9.7 & 39,873 & 9.1 & 2,483 & 11.8 & 680 & 9.7 & 43,036 \\
\hline North Carolina & 7.2 & 11,365 & 8.0 & 4,356 & 9.4 & 891 & 7.5 & 16,612 \\
\hline North Dakota & 16.2 & 1,112 & 10.9 & 201 & 15.0 & 840 & 15.0 & 2,153 \\
\hline Ohio & 10.8 & 24,024 & 7.7 & 3,652 & 6.2 & 351 & 10.2 & 28,027 \\
\hline Oklahoma & 20.7 & 12,142 & 18.4 & 3,182 & 22.1 & 3,076 & 20.5 & 18,400 \\
\hline Oregon & 16.5 & 11,205 & 19.7 & 2,363 & 21.7 & 1,421 & 17.4 & 14,989 \\
\hline Pennsylvania & 12.4 & 29,441 & 11.2 & 4,220 & 9.2 & 367 & 12.2 & 34,028 \\
\hline Rhode Island & 19.2 & 4,452 & NA & - & NA & - & 19.2 & 4,452 \\
\hline South Carolina & 8.7 & 7,015 & 6.4 & 1,344 & 4.9 & 190 & 8.1 & 8,549 \\
\hline South Dakota & 14.8 & 1,376 & 4.5 & 87 & 17.7 & 1,510 & 15.1 & 2,973 \\
\hline Tennessee & 9.2 & 10,102 & 12.9 & 3,958 & 10.2 & 660 & 10.0 & 14,720 \\
\hline Texas & 6.6 & 38,839 & 10.1 & 5,200 & 9.0 & 1,945 & 7.0 & 45,984 \\
\hline Utah & 5.0 & 3,739 & 5.9 & 288 & 12.7 & 480 & 5.4 & 4,507 \\
\hline Vermont & 16.0 & 741 & 14.4 & 598 & 14.4 & 628 & 15.0 & 1,967 \\
\hline Virginia & 5.5 & 8,757 & 9.0 & 1,512 & 15.3 & 912 & 6.1 & 11,181 \\
\hline Washington & 10.8 & 14,619 & 15.1 & 2,364 & 17.3 & 374 & 11.3 & 17,357 \\
\hline West Virginia & 16.1 & 3,503 & 19.5 & 1,876 & 21.7 & 1,580 & 18.0 & 6,959 \\
\hline Wisconsin & 9.5 & 9,367 & 7.5 & 2,265 & 10.2 & 356 & 9.1 & 11,988 \\
\hline Wyoming & 21.3 & 847 & 15.3 & 138 & 16.7 & 1,289 & 18.0 & 2,274 \\
\hline
\end{tabular}

Shading: States with nonmetro adjacent or remote rural counties with higher rates than metro counties 